\title{
Detection of an orbiting gas disk in the Red Rectangle ${ }^{\star}$
}

\author{
V. Bujarrabal ${ }^{1}$, R. Neri ${ }^{2}$, J. Alcolea ${ }^{3}$, and C. Kahane ${ }^{4}$ \\ 1 Observatorio Astronómico Nacional, Apartado 1143, 28800 Alcalá de Henares, Spain \\ 2 IRAM, 300 rue de la Piscine, 38406 St Martin d'Hères, France \\ e-mail: neri@iram.fr \\ 3 Observatorio Astronómico Nacional, C/Alfonso XII, 3, 28014 Madrid, Spain \\ e-mail: j .alcolea@oan.es \\ ${ }^{4}$ Laboratoire d'Astrophysique de Grenoble, BP 53, 38041 Grenoble Cedex 9, France \\ e-mail: Claudine.Kahane@obs.ujf-grenoble.fr
}

Received 9 April 2003 / Accepted 19 June 2003

\begin{abstract}
We present interferometric maps of $\mathrm{CO}$ emission in the Red Rectangle, a well known protoplanetary nebula. The $\mathrm{CO}$ emission is found to arise from a relatively thin equatorial disk, extending about $5^{\prime \prime}$ in the direction perpendicular to the symmetry axis of the optical nebula. The velocity dispersion of the emission clearly increases towards the center, in a pattern significantly coincident with that expected for a Keplerian velocity field. Modeling of the CO maps confirms that the emitting gas is probably rotating around the central star(s), with a Kepler-like velocity distribution (at least in the central regions) that would correspond to a central mass $\sim 0.9 M_{\odot}$. Other possible explanations to the observations are discussed, but are found to be unlikely. Our models also suggest that the density and temperature increase towards the center roughly proportionally to the inverse radius. The asymmetry observed in the line profile and intensity distribution (the red part being stronger) can be explained by self-absorption if, superimposed to the rotation velocity, there is a low radial expansion at a velocity of about $0.4 \mathrm{~km} \mathrm{~s}^{-1}$, at least in the outer disk regions. This is the first probable detection of a gas disk in Kepler-like rotation around a post-AGB star.
\end{abstract}

Key words. stars: AGB and post-AGB - stars: circumstellar matter - radio-lines: stars planetary nebulae: individual: Red Rectangle

\section{Introduction}

The existence of gas disks orbiting around post-AGB stars has been postulated several times (often associated with the presence of a stellar or substellar companion), in order to explain the very energetic axial flows that take place in this evolutionary phase (Soker 2002; Frank et al. 2003, and references therein). Such bipolar flows are very important in the evolution of protoplanetary nebulae (PPNe), particularly because they are expected to yield the axisymmetric shapes very often observed in PNe. Whether these bipolar post-AGB jets result from accretion via a rotating disk is a basic question. Such a process seems necessary to explain the very high linear momenta and kinetic energy observed in post-AGB bipolar flows (Bujarrabal et al. 2001), since radiation pressure as the driving mechanism is very probably ruled out by the observational data.

Send offprint requests to: V. Bujarrabal,

e-mail: v.bujarrabal@oan.es

* Based on observations carried out with the IRAM Plateau de Bure Interferometer. IRAM is supported by INSU/CNRS (France), MPG (Germany) and IGN (Spain).
Disks/tori of molecular gas around post-AGB stars are commonly detected as the central part of protoplanetary nebulae, though they are not observed to rotate, but to be systematically in expansion (as the rest of the nebula); see the cases of M 1-92 (Bujarrabal et al. 1998), M2-9 (Zweigle et al. 1997), M2-56 (Castro-Carrizo et al. 2002), etc. Only in $\mathrm{OH} 231.8+4.2$, VLBI observations of $\mathrm{SiO}$ maser emission show the presence of a number of spots, surrounding the star at a few $\mathrm{AU}$, at velocities that are compatible with rotation (Sánchez Contreras et al. 2002). But the few spots detected and the difficult interpretation of the maser data prevented any definitive conclusion.

The Red Rectangle is a well known PPN, surrounding the A1 star HD 44179, a spectroscopic binary (e.g. Waelkens et al. 1996). The optical nebula is very extended (about $1^{\prime}$ ) and presents a conspicuous axis of symmetry at position angle $\sim 11^{\circ}$. Jura et al. $(1995,1997)$ proposed the presence of orbiting material in the Red Rectangle, in order to explain its peculiar molecular line profiles (compatible with the emission from a rotating disk) and the probable presence of big grains in it, whose formation would need a long-lived (i.e. 
not rapidly expanding) reservoir of grains. The existence of a long-lived disk around HD 44179 has been also proposed to explain the anomalous abundances found in the stellar atmosphere, in which refractory elements are severely underabundant, see e.g. Waelkens et al. (1996). Following similar arguments, disks have been also proposed for a few other evolved stars, see Waters et al. (1992), Jura \& Kahane (1999), etc.

However, such disks had not been observed to date, their very existence remained to be demonstrated and their main properties to be studied. In this paper we present the first probable detection of a disk with Kepler-like rotation in the Red Rectangle, and the first at all in a PPN. Our CO mapping of this nebula shows the presence of a gas disk perpendicular to the symmetry axis of the nebula, whose velocity distribution undoubtedly presents the signatures of rotation, with a velocity distribution close to that expected for Keplerian orbiting.

\section{Observations}

We have observed the Red Rectangle in the CD set of configurations of the five-antenna IRAM array, at Plateau de Bure, between December 1997 and October 1999. Projected baselines range from about $170 \mathrm{~m}$ down to the antenna diameter of $15 \mathrm{~m}$. The primary beam width is $42^{\prime \prime}$ at $115 \mathrm{GHz}$ and $21^{\prime \prime}$ at $230 \mathrm{GHz}$.

At $1.3 \mathrm{~mm}$, data were taken in double sideband mode with the receivers tuned to $230.538 \mathrm{GHz}$ (lower sideband). At $2.6 \mathrm{~mm}$, observations were made in upper sideband only, with the SIS receivers tuned to $115.2712 \mathrm{GHz}$. One correlator unit of $20 \mathrm{MHz}$ bandwidth spanning 256 channels was devoted to the $\mathrm{CO} J=2-1$ line centered at the systemic velocity of $1 \mathrm{~km} \mathrm{~s}^{-1}$. A second unit of $10 \mathrm{MHz}$ bandwidth was devoted to the CO $J=1-0$ line to provide the same velocity resolution. This setup that gave a spacing between channels of $0.1 \mathrm{~km} \mathrm{~s}^{-1}$ was used to produce visibility tables with an effective velocity resolution of $\sim 0.2 \mathrm{~km} \mathrm{~s}^{-1}$. Four additional correlator units, each with a $160 \mathrm{MHz}$ bandwidth, were devoted to observe continuum emission near the line frequencies. Visibilities were obtained using on-source integration times of $20 \mathrm{~min}$ interspersed with 4 min calibrations on the continuum sources 0607-157 and 0607-083. Atmospheric phase noise was typically between 5 and 40 degrees at $2.6 \mathrm{~mm}$ (a factor of two higher at $1.3 \mathrm{~mm}$ ). Only visibilities consistent with seeing conditions better than $11^{\prime \prime} 0$ were retained. The absolute flux density scale, which was established with observations of MWC 349 (1.12 Jy at $2.6 \mathrm{~mm}, 1.70 \mathrm{Jy}$ at $1.3 \mathrm{~mm})$, is in full agreement with the interferometric efficiency and should be accurate to $10 \%$ at $2.6 \mathrm{~mm}$ and to about $20 \%$ at $1.3 \mathrm{~mm}$. The instrumental passband shape was determined on the quasars 3C 454.3 and 0923+392. Data calibration was carried out in the antenna-based mode to obtain the time-dependent complex gains of each antenna. Channel maps were produced with a velocity resolution of $\sim 0.6 \mathrm{~km} \mathrm{~s}^{-1}$ providing a uniformly weighted synthesized half-power beam width of 5 ." $^{\prime \prime} \times 2$." 7 (the major axis being oriented at PA $=6^{\circ}$ ) at the frequency of the ${ }^{12} \mathrm{CO} J=1-0$ transition, and of $3 .^{\prime \prime} 0 \times 11^{\prime \prime} 6\left(\mathrm{PA}=-25^{\circ}\right)$ at the frequency of the ${ }^{12} \mathrm{CO} J=2-1$ transition. We measured a mean point source sensitivity noise limit of $20 \mathrm{mJy}^{\text {beam }}{ }^{-1}$
$(1 \sigma)$ in channels of $0.6 \mathrm{~km} \mathrm{~s}^{-1}$ at $115.2712 \mathrm{GHz}$, and a sensitivity of $35 \mathrm{mJy}_{\text {beam }}^{-1}$ at $230.538 \mathrm{GHz}$, respectively corresponding to an rms temperature of $150 \mathrm{mK}$ and $160 \mathrm{mK}$ (in units of $T_{\mathrm{mb}}$, main-beam Rayleigh-Jeans-equivalent antenna temperature); fully consistent at both frequencies with the effective integration times and mean system temperatures. The conversion factors to the main-beam temperature scale are $7.2 \mathrm{~K} / \mathrm{Jy}$ beam $^{-1}$ at $2.6 \mathrm{~mm}$, and $4.8 \mathrm{~K} / \mathrm{Jy}_{\text {beam }}{ }^{-1}$ at $1.3 \mathrm{~mm}$.

We have checked that the angle integrated line profiles of both $J=1-0$ and $J=2-1$ lines are consistent in shape and intensity with the single-dish spectra by Jura et al. (1995) within $20 \%$, which is comparable to the observational uncertainties. So, possible flux loss in the interferometric data is negligible.

Continuum visibility records were computed from linefree channels that spanned nearly $280 \mathrm{MHz}$ at both $1 \mathrm{~mm}$ and $3 \mathrm{~mm}$ bands. In the resulting maps, the continuum sensitivity $(5 \times \mathrm{rms})$ is $35 \mathrm{mJy}^{-1}$ beam ${ }^{-1}$ at $2.6 \mathrm{~mm}$ and $25 \mathrm{mJy}^{-1}$ beam ${ }^{-1}$ at $1.3 \mathrm{~mm}$. The deconvolved continuum emission at $1.3 \mathrm{~mm}$ can be approximately described by a Gaussian function with major and minor full widths at half maximum of $1 . \prime 7$ and $0 . ' 4$, oriented at $36^{\circ}$ (position angle of major axis). Due to the limited sensitivity of our mapping of the continuum emission, the possible flux loss at this frequency and the small observed extent of the continuum brightness distribution, its shape is only poorly measured. No extent of the $2.6 \mathrm{~mm}$ continuum could be measured. The total intensity is equal to $200 \mathrm{mJy}$ at $1.3 \mathrm{~mm}$, and to $32 \mathrm{mJy}$ at $2.6 \mathrm{~mm}$.

Our observational results are presented in Figs. 1 and 2. In Fig. 1 we show the spatial distribution of the $\mathrm{CO} J=2-1$ transition per velocity channels; the continuum contribution has not been subtracted. In this figure we also show the $1.3 \mathrm{~mm}$ continuum distribution and the dirty and clean synthesized beams. In Fig. 2 we show the velocity-position diagrams along the direction perpendicular to the optical axis of the nebula of both $J=1-0$ and $J=2-1$ transitions (together with our model predictions, see Sect. 3.2). In our presentation of the observations, we have always adopted the centroid of the continuum source $\left(\alpha_{\mathrm{J} 2000}=06^{\mathrm{h}} 19^{\mathrm{m}} 58^{\mathrm{s}} .216\right.$ and $\left.\delta_{\mathrm{J} 2000}=-10^{\prime \prime} 38^{\prime} 14^{\prime \prime} .58\right)$ as the spatial origin.

\section{Results}

\subsection{The distribution of the $\mathrm{CO}$ emission}

In Fig. 1 we show the maps per velocity of the CO $J=2-1$ transition. As we see the spatial total extent is not very large compared to the telescope resolution. However, we clearly find that $\mathrm{CO}$ emission is extended in the direction perpendicular to the optical nebula axis (whose position angle is $11^{\circ}$, see Sects. 1 and 3.2), with a total extent $\sim 5^{\prime \prime}$; we note that the brightness distribution is particularly wide in the low-velocity channels. We only measure a limit to the extent in the axial direction, which must be smaller than $\sim 1^{\prime \prime}$. We clearly identify a variation with the velocity of the emission centroids, along the direction perpendicular to the optical nebula axis. The centroid positions become positive or negative depending on the sign of the velocity shift with respect to the line center; such 
The Red Rectangle

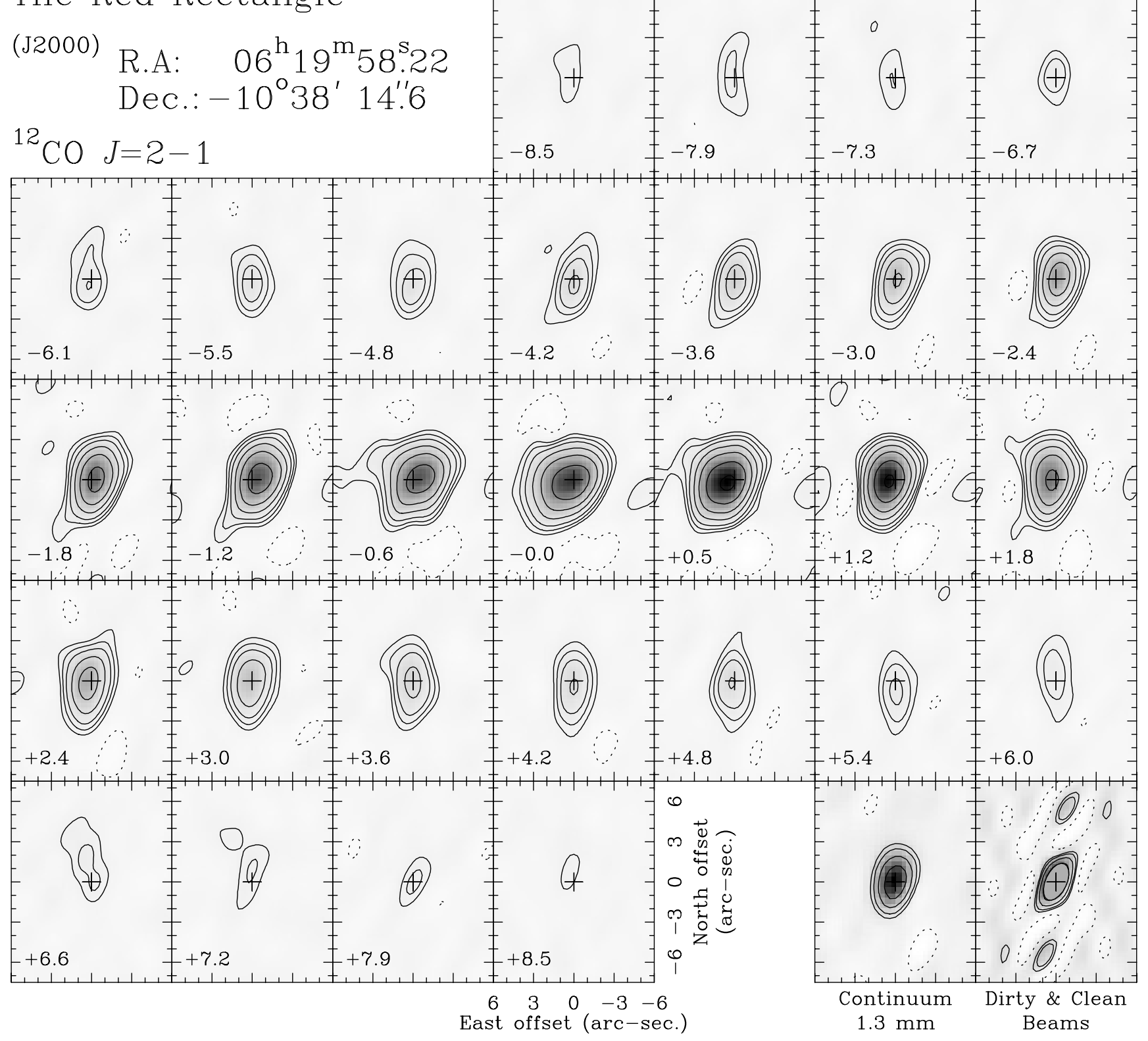

Fig. 1. Plateau de Bure maps of the Red Rectangle in CO $J=2-1$ for a velocity resolution of $0.6 \mathrm{~km} \mathrm{~s}^{-1}$ (continuum not subtracted). The central LSR velocity (in $\mathrm{km} \mathrm{s}^{-1}$ ) of each channel map is indicated in the bottom left corner. The contours are logarithmically spaced (4 contours per decade): $-0.10,0.10,0.18,0.30,0.56,1.0,1.8$ and $3.0 \mathrm{Jy} \mathrm{beam}^{-1}$ (equivalent to $-0.48,0.48,0.86,1.4,2.7,4.8,8.6$ and $14.4 \mathrm{~K}$ in $T_{\mathrm{mb}}$ units). Negative contours are indicated with dotted lines. We also present the continuum map at $\lambda=1.3 \mathrm{~mm}$ (contours: $-18,18,30,56$ and $100 \mathrm{mJy}$ beam $\left.^{-1}\right)$, and the dirty and clean beams $(-9,9,15$ and $28 \%$ levels of the dirty beam, plus the $50 \%$ contour of the clean beam).

spatial separation is clearly the highest for small velocity shifts (in absolute value). In Fig. 2 we show the corresponding velocity-position distribution along the equatorial direction, defined as being perpendicular to the symmetry axis of the optical nebula. We do not show the maps per velocity of the CO $J=1-0$ line, because of the poor resolution at its frequency; an equatorial cut is shown in Fig. 2.

As we see in Fig. 2, the CO velocity-position distribution along the nebula equator is very similar to that expected from a Kepler-like rotating disk. This distribution strongly suggests rotation around the nebula axis, excluding any strong radial expansion (see also Sect. 3.2). No trace is found in the Red Rectangle of an extended molecular envelope similar to those usually found around AGB and post-AGB stars (we recall at this respect that no sign of flux loss with respect to single-dish profiles is found, Sect. 2). The kinematics of the detected disk is also significantly different to the relatively fast expansion characteristic of AGB envelopes and of other disks found in PPNe (Sect. 1).

\subsection{Modeling}

We have modeled our data using calculations of CO line emission similar to those presented in Bujarrabal et al. (1997, 1998), which predict maps of $\mathrm{CO}$ emission per velocity and velocityposition cuts along different directions. In these calculations, 


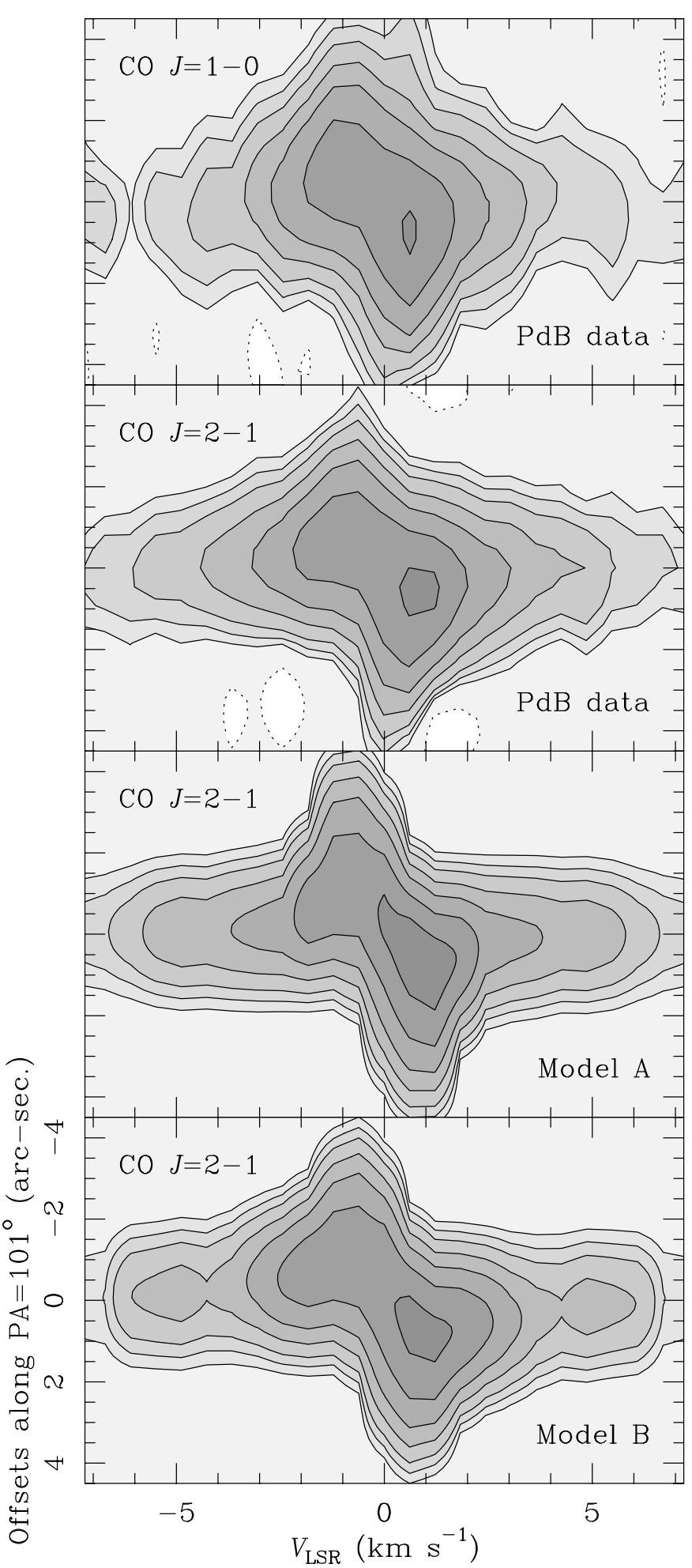

Fig. 2. Velocity-position diagrams along the direction perpendicular to the axis of the optical image of the nebula (i.e. at position angle equal to $101^{\circ}$ ). The lowest two panels show our theoretical predictions for models A and B (see text). For the $J=2-1$ line data and models, contours are as in Fig. 1, i.e.: -0.10, 0.10, 0.18, 0.30, 0.56, 1.0, 1.8 and $3.0 \mathrm{Jy}^{\text {beam }}{ }^{-1}$ (equivalent to $-0.48,0.48,0.86,1.4,2.7$, $4.8,8.6$ and $14.4 \mathrm{~K}$ in $T_{\mathrm{mb}}$ units). For the $J=1-0$ line, contours are $-0.03,0.03,0.06,0.10,0.18,0.30,0.58$ and $1.0 \mathrm{Jy} \mathrm{beam}^{-1}$ (equivalent to $-0.2,0.2,0.4,0.7,1.3,2.2,4.0$ and $7.2 \mathrm{~K}$ in $T_{\mathrm{mb}}$ units). Negative contours are indicated with dotted lines. Continuum emission has not been subtracted. the $\mathrm{CO}$ excitation is described by a rotational temperature, which we will assume to be similar to the kinetic temperature (see discussion in the above papers). Both density and velocity can vary from point to point inside the model nebula. Radiative interaction between the different nebular regions, including opacity effects, is taken into account; in the present case we assumed a local velocity dispersion equal to that given by the thermal broadening.

Since the geometry of the region emitting in the continuum is not well known and, in any case, it is probably significantly more extended than the regions emitting in the line wings (the only velocities at which the contribution of the weak continuum in the maps can be noticeable), we have accounted for the continuum in our model just adding its observed distribution to those predicted for the lines.

In our models we will assume the value of the distance adopted in our previous works, $D \sim 380 \mathrm{pc}$, similar to that used by Cohen et al. (1975) and compatible with the uncertain Hipparcos parallax. Men'shchikov et al. (2002) deduced a distance $\sim 710 \mathrm{pc}$ from their fitting of the light scattered and emitted by dust. We will still adopt the shorter distance, though uncertain, to ease the comparison with previous works and since the distance value from the dust continuum fitting can also be affected by the uncertainties in the model, particularly in the grain size and optical properties. See further discussion favoring short distances in Jura et al. (1997). In any case, our model parameters can be easily scaled to other values of $D$ : the central mass and the nebula linear size would vary proportionally to the distance, the density is inversely proportional to $D$, and the total molecular nebula mass would vary as $D^{2}$.

The values of the inclination of the nebula axis of symmetry with respect to the plane of the sky, derived from scattered light images, vary between $\sim 0^{\circ}$ and $10^{\circ}$ (Roddier et al. 1995; Lopez et al. 1995; Men'shchikov et al. 2002), with the southern lobe pointing to us. We will choose an intermediate value, $\sim 5^{\circ}$, which yields a good fitting of our $\mathrm{CO}$ observations. Inclinations equal to $0^{\circ}$ or $10^{\circ}$ have been also investigated, but the fitting is in general slightly less satisfactory. From the existing images, the inclination of the nebula axis with respect to the north direction (to the east, i.e. the position angle) is deduced to be $\sim 11^{\circ}$.

In our models we will assume that the $\mathrm{CO}$ emission comes from a disk perpendicular to the nebula axis (as implied by the observed image dimensions). The disk is defined by its radius and thickness. The main velocity field in the disk is assumed to be rotation around the axis, but other kinematical features can also be included.

In order to obtain the observed asymmetry in velocities (as due to self-absorption, see below) and to explain the relatively strong intensity of the high velocity features (that must come from a tiny inner region in the case of Keplerian rotation), the temperature and density must decrease with radius, $r$. We considered variations of the density $n \propto 1 / r^{\alpha_{n}}$, with $\alpha_{n}$ between 1 and 2 . For the temperature we assumed variations similar to those found for AGB envelopes (Goldreich \& Scoville 1976; Groenewegen et al. 1998; Schöier \& Olofsson 2001, etc.), $T \propto 1 / r^{\alpha_{T}}$ with $\alpha_{T}$ ranging between 0.7 and 1 , and $T \sim 1000 \mathrm{~K}$ in the central regions and $\sim 30-100 \mathrm{~K}$ at $10^{16} \mathrm{~cm}$. 
The fitting strategy has been, first, to determine the geometrical and kinematical parameters; the kinematics is relatively independent of the rest of the model and quite accurately measured. Second, the density and temperature are fitted. The final fitting has been adopted after an iterative procedure, which includes reconsideration of the geometry and velocity field, etc.

A first fitting of the model parameters is given in Table 1. The predicted velocity-position diagram (the most significant result to be fitted by the calculations) is shown in Fig. 2 (model A), to be compared with observations in the same figure. The results are also compatible with the data shown in Fig. 1 and in Fig. 2 for the $J=1-0$ transition. As we see, the quality of the fitting is quite satisfactory, mostly in view of the simplicity of the nebula model. We have tested the quality of the fitting by calculating the visibilities in the $u v$ plane corresponding to the model predictions, the difference with respect to the observations and, finally, the corresponding differential image. In general, the differences between the model predictions and the observations given by this procedure are $\sim 10-20 \%$, which can be considered as satisfactory.

However, the determination of the model parameters from the fitting process is not always accurate. The first problem is that the $\mathrm{CO}$ emission is optically thick, leading uncertain determinations of the density and total mass. A high optical depth is necessary to explain the relatively high brightness temperatures measured (in spite of the large synthesized beams) and the relatively strong $J=1-0$ line, which for high temperatures and low opacities must be about 4 times weaker than the $J=2-1$ one. This factor should still be significantly increased by the fact that the beam in the 1-0 transition is relatively large. It is clear that the density must increase towards the center, to yield high opacities even at high velocities (that come from small regions, due to the velocity law assumed). In general, a more accurate estimate of the density distribution and total mass would require observations of ${ }^{13} \mathrm{CO}$ or even $\mathrm{C}^{18} \mathrm{O}$ lines. For a ${ }^{12} \mathrm{CO}$ relative abundance equal to $2 \times 10^{-4}$ (Bujarrabal et al. 2001), we derive a relatively uncertain value of the total mass of the molecular gas $\sim 0.04 M_{\odot}$.

The temperature is also difficult to measure, since the line intensity (in this optically thick case) depends not only on the temperature but also on the dilution factor in the beam, which is largely unknown (since the disk thickness is not well measured from our data, see below). For that reason we used quite strong constraints to the temperature law from results on AGB envelopes. Most of the information from the fitting on this law comes from the fact that we must explain the relatively high intensity measured at high velocities, and that we have tried to explain the velocity asymmetry of the emission by means of absorption by the outer layers. We can deduce that the uncertainty in the gas temperature is of about a factor 2, since the disk thickness cannot increase by more than this factor (to avoid contradiction with observations, as explained below) and gas temperatures larger than twice those given here would be too high with respect to those expected in AGB envelopes.

The outer diameter of the disk is well defined by the measured size in the equatorial direction. For the distribution of $\mathrm{CO}$ emissivity assumed here, the value given in the table is accurate at $\pm 10 \%$ level; from the total spatial dispersion of the data, and
Table 1. Structure and physical conditions in the molecular disk in the Red Rectangle, derived from our simplified model fitting of the $\mathrm{CO}$ data (model A). Other parameters assumed in our modeling are also given.

\begin{tabular}{|c|c|c|}
\hline Free parameters & Law & Fitted values \\
\hline Disk outer radius & constant & $\begin{array}{l}1.5 \times 10^{16} \mathrm{~cm} \\
\left(2^{\prime \prime} 6 \text { for } D=380 \mathrm{pc}\right) \\
\end{array}$ \\
\hline Disk thickness & constant & $5 \times 10^{15} \mathrm{~cm}$ \\
\hline Temperature & $T \propto 1 / r^{\alpha_{T}}$ & $\begin{array}{l}T\left(10^{16} \mathrm{~cm}\right)=40 \mathrm{~K} \\
\alpha_{T}=0.8\end{array}$ \\
\hline Gas density & $n \propto 1 / r^{\alpha_{n}}$ & $\begin{array}{l}n\left(10^{16} \mathrm{~cm}\right)=6 \times 10^{6} \mathrm{~cm}^{-3} \\
\alpha_{n}=1.1\end{array}$ \\
\hline $\begin{array}{l}\text { Tangential } \\
\text { velocity }\end{array}$ & $\begin{array}{l}V_{t} \propto 1 / \sqrt{r} \\
(\text { Keplerian }) \\
\end{array}$ & $\begin{array}{l}V_{t}\left(10^{16} \mathrm{~cm}\right)=1 \mathrm{~km} \mathrm{~s}^{-1} \\
\left(M_{*}=0.9 M_{\odot}\right)\end{array}$ \\
\hline $\begin{array}{l}\text { Expansion } \\
\text { velocity }\end{array}$ & constant & $V_{e}=0.4 \mathrm{~km} \mathrm{~s}^{-1}$ \\
\hline \multicolumn{2}{|l|}{ Fixed parameters } & Assumed values \\
\hline \multicolumn{2}{|l|}{ Distance } & $380 \mathrm{pc}$ \\
\hline \multicolumn{2}{|c|}{$\begin{array}{l}\text { Axis inclination } \\
\text { from the plane of the sky }\end{array}$} & $5^{\circ}$ \\
\hline \multicolumn{2}{|c|}{$\begin{array}{l}\text { Axis inclination } \\
\text { in the plane of the sky (PA) }\end{array}$} & $11^{\circ}$ \\
\hline \multicolumn{2}{|c|}{ CO relative abundance } & $2 \times 10^{-4}$ \\
\hline
\end{tabular}

taken into account the beam width, we think that equatorial diameters significantly smaller than 4 " are very improbable. On the other hand, the low extent measured in the axial direction suggests that the disk thickness is small and prevents an accurate measurement of this parameter. All we can say is that it is smaller than $\sim 10^{16} \mathrm{~cm}$. We took a value of $5 \times 10^{15} \mathrm{~cm}$, since much smaller values yield (for the general model properties used here) too high kinetic temperatures, compared to the possible laws discussed above. However, we note the uncertain lower limit to the disk thickness, due to the many possible combinations of temperature and density laws (including in particular densities decreasing with the distance to the equatorial plane, for which the definition of "thickness" will even be different; such details cannot be studied from our data due to their moderate resolution, limited mapping sensitivity and the high optical depth of the $\mathrm{CO}$ emission). 
It is difficult to measure from our calculations the inner radius of the molecular rotating disk, due to the moderate spatial resolution. But we can deduce, from the high intensity observed at velocities $\gtrsim 5 \mathrm{~km} \mathrm{~s}^{-1}$ with respect to the systemic velocity and assuming Keplerian rotation (see below), that molecular gas at a temperature $\sim 500 \mathrm{~K}$ must exist (and be opaque in ${ }^{12} \mathrm{CO}$ $J=2-1)$ at distances as short as $\sim 5 \times 10^{14} \mathrm{~cm}$ from the star. We note that this radius implies a typical angular diameter of about 0 '. 17 for the inner $\mathrm{CO}$ regions, in agreement with the very small separation found (Fig. 2) between the regions emitting at both extreme velocities $\left(<0{ }^{\prime \prime} 25\right)$. To study the $\mathrm{CO}$ emission inside this limit, observations with higher spatial resolution and sensitivity would be needed.

The velocity field is relatively well measured. We find that the observations can be explained by Kepler-like rotation for a central mass of $0.9 M_{\odot}$. As we see in the figures, the red part of the observed CO spectra is significantly more intense than the blue one. This can be explained assuming the presence of self-absorption, provided that the temperature decreases with the distance from the center and there is some expansion (at least in the outer layers); a similar phenomenon is observed in many AGB envelopes and some PPNe. For the temperature profile described a few paragraphs above and in Table 1, an expansion velocity just $\sim 0.4 \mathrm{~km} \mathrm{~s}^{-1}$ reasonably reproduces the observed velocity asymmetry. The presence of much larger expansion velocities yields rounded structures in the synthetic position-velocity diagrams that are not observed; predictions of models including expansion movements in the whole nebula faster than $1 \mathrm{~km} \mathrm{~s}^{-1}$ are in contradiction with the observations. Note that this severe self-absorption becomes very strongly dependent on the temperature and velocity laws; therefore, further fitting of such details of our maps is, in our opinion, not relevant. On the other hand, it is obvious that the observed profile asymmetry could be also explained by a more complex dependence of the density/temperature distributions on the radius and the azimuthal angle (around the nebula axis), but we will adopt the self-absorption explanation as intuitively requiring less hypotheses. This low expansion velocity indicates that the CO disk would have a large kinematic age, $\gtrsim 10^{4} \mathrm{yr}$, much larger than the usually assumed duration of the post-AGB phase, $\sim 1000 \mathrm{yr}$.

It is important to note that the observed velocity distribution could also be explained by (improbable) velocity fields, other than orbiting dominated ones. The high-velocity emission could be due to outward fast jets (which are often present in PPNe). However, such jets would be in the Red Rectangle quite different from those observed in other nebulae:

1) The jets should be almost exactly perpendicular to the nebula axis. In almost all well studied PPNe and PNe the situation is the opposite, with fast molecular jets being closely parallel to the axis, plus an equatorial torus or disk expanding at low velocity (see data compilation and references in Bujarrabal et al. 2001). Only in two objects, CRL 2688 (one component) and Frosty Leo, molecular jets appear at angles well distant from the axis. In CRL 2688 (Cox et al. 2000), one of the CO jets is almost perpendicular to the axis, but in that nebula several other jets appear and the equatorial one only carries a small fraction of the high velocity material (Bujarrabal et al. 2001). In
Frosty Leo (Castro-Carrizo et al. 2003), the dense molecular jet forms an angle of about $45^{\circ}$ with respect to the obvious nebula axis, being therefore not so close to the equator.

2) If the velocity-position structure observed in the $\mathrm{CO}$ lines from the Red Rectangle is explained by outflowing kinematics, the highest velocities should occur in the innermost jet regions, contrary to what is found in all mapped PPNe (see references in Bujarrabal et al. 2001). In fact, the outflow velocity significantly increases with the distance to the star along the $\mathrm{CO}$ emitting jets of most of these nebulae, including CRL 2688 and Frosty Leo. The $\mathrm{OH}$ maser observations in PPNe by Zijlstra et al. (2001) also reveal systematically positive velocity gradients in the fast outflowing gas. Moreover, even if the (improbable) equatorial outflows in the Red Rectangle show an ad hoc velocity law $\underset{\sim}{\sim} \sqrt{1 / r}$, as the Keplerian tangential velocity, we would had expected a clear central minimum of the brightness distribution at the systemic velocity, again in contradiction with observations. We can see in the case of the complex jet structure of CRL2688 (Cox et al. 2000) that a combination of independent jets in general will not lead to velocity-position diagrams similar to those observed in the Red Rectangle.

We therefore conclude that the explanation of the observed velocity distribution by means of outflows will require very unexpected and ad hoc properties for such components. Rotation is by far the simplest explanation.

\subsubsection{Model B}

As we have seen before, our observations are in general well explained by our model A. But a few observational features are not well reproduced. Note first that the velocity extent of the low-velocity emission at the largest distance from the star is narrower in the observed velocity-position diagram (Fig. 2). Even using a local velocity dispersion just equal to the thermal dispersion, we cannot reproduce such a narrow feature. We think that this is due to the presence of negative side-lobes in the interferometric observations, not completely eliminated by the cleaning procedure (probably due to some residual error in the calibration of these long and complex observations, Sect. 2.1). In fact, a negative contour appears in our $J=2-1$ diagram (at the expected velocity, $V_{\mathrm{LSR}} \sim 1-2 \mathrm{~km} \mathrm{~s}^{-1}$ ), and a similar effect, but with the opposite sign, conspicuously appears in the diagram of the $J=1-0$ transition (Fig. 2). The negative lobes are expected to become visible precisely at the velocity of the intensity maxima, decreasing the extent of the discussed observational features. Residual negative lobes $\sim 10 \%$ of the peak could explain this effect.

Also note that the slope of the intensity curves in the upperright and lower-left quadrants is somewhat steeper in the model predictions (Fig. 2, model A). This can be due to that the actual variation with radius of the rotational velocity is not fully Keplerian, with a faster decrease of the velocity in the outer layers. Such a law is to be expected, in fact, since, if expansion exists, a Keplerian rotation should be substituted by a decrease proportional to $1 / r$, due to the angular momentum conservation. In Fig. 2 we also show the results from another model (model B), in which all physical parameters remain very 
similar to those adopted in model A (Table 1), except for that the expansion velocity only appears farther than $3 \times 10^{15} \mathrm{~cm}$ and the rotational velocity modulus satisfies $V_{t} \propto 1 / \sqrt{r}$ inside this limit and $V_{t} \propto 1 / r$ outwards. As we see, the fitting of the observations is somewhat better under this velocity field.

Further attempts to discriminate between both models and to study of the velocity field are meaningless, in our opinion, because of the limitations of the data.

\section{Discussion and conclusions}

We present maps of the CO $J=2-1$ and $J=1-0$ emission from the Red Rectangle, a well known protoplanetary nebula (PPN) surrounding the post-AGB star HD 44179. The molecular emission seems to come from a relatively thin disk perpendicular to the axis of symmetry of the optical nebula. The velocity field of this disk is well defined by the observations, being in good agreement with Keplerian orbiting around the central star(s).

We have performed model calculations using previously described codes (Sect. 3.2). The values found for the different parameters from our simplest model fitting (model A) and some assumed parameters are summarized in Table 1. Our calculations confirm that the dominant velocity field is that of rotation. At least in the inner regions, the rotation velocity law is probably Keplerian. On the other hand, the line profiles present a significant asymmetry, the red part being more intense. This feature is well explained by self-absorption assuming, together with rotation, a small expansion velocity $\left(\sim 0.4 \mathrm{~km} \mathrm{~s}^{-1}\right)$, at least in the outer regions of the disk (and provided that the temperature decreases with the radius). The best fitting of the data (model B, Fig. 2) is found assuming that Keplerian rotation only holds for the inner parts of the envelope, closer than about $3 \times 10^{15} \mathrm{~cm}$, where expansion is not necessarily significant. At longer distances, the gas would still be rotating, but the slow expansion must be present and the tangential velocity modulus would decrease faster, $V_{t} \propto 1 / r$. This velocity field is coincident with that given by the law of conservation of the angular momentum in a rotating gas that is also in expansion. The whole kinematics would then physically correspond to a Keplerian inner disk from which gas is escaping at low velocity; some gas evaporation process or radiation pressure would then be acting onto a relatively stable rotating disk. The fact that the sound velocity of the gas, and indeed its radial expansion velocity, are only somewhat smaller than the rotation velocity may explain why the disk thickness is not much smaller than its radius (e.g. Dominik et al. 2003; Chiang \& Goldreich 1997). Other significantly different velocity fields have been discussed and found to represent improbable or minor contributions to the whole movements.

The total central mass (in our standard model) is found to be $\sim 0.9 M_{\odot}$, for the Keplerian velocity field discussed above. We note that this value is larger than the mass of each of the stars in the center of the model disk by Men'shchikov et al. (2002), but in good agreement with the sum of both stellar masses (Men'shchikov et al. deduced the existence of two central stars, with masses equal to 0.35 and $0.57 M_{\odot}$ ). We note that in Men'shchikov et al.'s model there is additional $1 M_{\odot}$ of circumstellar material very close to the star. The discrepancy between the total central mass obtained in that paper and from our data could be mainly due to the different distance assumed (Sect. 3.2).

We derive a total mass of the disk $\sim 0.04 M_{\odot}$, but our estimation is not accurate, since the line emission is optically thick in most velocities and lines of sight. This value is almost ten times smaller than that deduced by Men'shchikov et al. (2002) from dust continuum fitting (after correction for the difference between the adopted distance values). However, in that model $90 \%$ of the mass is sharply concentrated in an inner torus, closer than $5-10 \times 10^{14} \mathrm{~cm}$ and with extremely high densities, $\sim 2.5 \times 10^{12} \mathrm{~cm}^{-3}$, at least one order of magnitude higher than the densities found in other nebulae around AGB or post-AGB stars. This very compact component is very different from the relatively extended one probed by our data. Our mass value is compatible with the lower limit estimated by Jura et al. (1997), from data on dust emission at long wavelengths. The disk radius deduced by Jura et al., between $3 \times 10^{15}$ and $2 \times 10^{16} \mathrm{~cm}$, is also compatible with the radius we measure from the emission of the molecular gas, $1.5 \times 10^{16} \mathrm{~cm}$ $\left(3 \times 10^{15} \mathrm{~cm}\right.$ for the inner Keplerian disk in model B).

From the relatively high intensity of the $\mathrm{CO}$ emission at absolute values of the relative velocity larger than $5 \mathrm{kms}$, and for the velocity law mentioned above, we deduce that molecular gas must exist at distances as short as $\sim 5 \times 10^{14} \mathrm{~cm}$ from the star. This typical inner radius is only about twice larger than the inner radius deduced by Men'shchikov et al. (2002), equivalent to about 40 mas; these authors derive this small value in order to explain the high-resolution images by Tuthill et al. (2002), which show that the light collimation takes place very close to the star. It is therefore very probable that dense material is present in the inner disk of the Red Rectangle down to a distance of this order.

The small extent of the $\mathrm{CO}$ emission in the direction of the optical nebular axis indicates that the disk is relatively thin, thinner than about $10^{16} \mathrm{~cm}$. We propose a value $\sim 5 \times 10^{15} \mathrm{~cm}$, which keeps the gas temperature at values not excessively high (by comparison with expectations, see Sect. 3.2) and avoids any conflict with observations.

The gas temperature law in the disk deduced from our model fitting is similar to those found in AGB envelopes, decreasing from $\sim 400 \mathrm{~K}$, at $5 \times 10^{14} \mathrm{~cm}$ from the center, to about $40 \mathrm{~K}$, at $10^{16} \mathrm{~cm}$. This parameter is also not very well constrained from our data, since its value depends on the assumed disk thickness (as mentioned above); we deduce an uncertainty for our temperature estimations of about a factor 2 .

As we have seen, our observations have allowed a number of important conclusions, particularly on the rotation properties of the disk, but are not good enough to accurately measure other parameters. This is mainly the case of the disk inner radius and thickness; the density and temperature distributions were also not well measured. To improve our knowledge on these parameters, future observations should improve at least the spatial resolution (the Plateau de Bure telescope can now reach resolutions better than $1^{\prime \prime}$ ) and the sensitivity (a noise level $\sim 2$ times smaller than that in our data, in $\mathrm{mJy} /$ beam, can be now attained). In particular, the expected shape and 
extent (deduced from our data and from optical-IR images, see Men'shchikov et al. 2002; Tuthill et al. 2002) of the inner nebular regions suggest that a moderate improvement in the resolution should allow mapping of the central (probably biconical) hole and measuring of the disk thickness and its variations with the radius (perhaps increasing outwards).

Finally, we note that, from the point of view of mass, dynamics, size and temperature, the rotating disk we have detected in the Red Rectangle is amazingly similar to disks found to orbit around forming stars (see e.g. Mannings et al. 2000), in spite of the uncertain values derived here for some parameters and the variations found in the properties of the disks around young stars. This result is particularly striking in view of the very different nature of the Red Rectangle nebula, an evolved post-AGB object, and of the disks associated with star formation, through which the young star accretes interstellar material. This accretion in young stars is thought to drive the ejection of bipolar flows, which carry the angular momentum excess of the rotating disks.

Acknowledgements. This work has been supported by the Spanish Ministerio de Ciencia y Tecnologia and European FEDER funds, under grants AYA2000-0927, ESP2001-4519-PE and ESP2002-01693.

\section{References}

Bujarrabal, V., Alcolea, J., Neri, R., \& Grewing, M. 1997, A\&A, 320, 540

Bujarrabal, V., Alcolea, J., \& Neri, R. 1998, ApJ, 504, 915

Bujarrabal, V., Castro-Carrizo, A., Alcolea, J., \& Sánchez Contreras, C. $2001, A \& A, 377,868$

Cohen, M., Anderson, C. M., Cowley, A., et al. 1975, ApJ, 196, 179

Castro-Carrizo, A., Bujarrabal, V., Sánchez Contreras, C., Alcolea, J., \& Neri, R. 2002, A\&A, 386, 633
Castro-Carrizo, A., et al. 2003, A\&A, in preparation

Chiang, E. I., \& Goldreich, P. 1997, ApJ, 490, 368

Cox, P., Lucas, R., Huggins, P. J., et al. 2002, A\&A, 353, L25

Dominik, C., Dullemond, C. P., Cami, J., \& van Winckel, H. 2003, A\&A, 397, 595

Frank, A., Blackman, E. G., Gardiner, T., \& Lery, T. 2003, ApJ, submitted

Goldreich, P., \& Scoville, N. 1976, ApJ, 205, 144

Groenewegen, M. A. T., Van der Veen, W. E. C. J., \& Matthews, H. E. 1998, A\&A, 338, 491

Jura, M., Balm, S. P., \& Kahane, C. 1995, ApJ, 453, 721

Jura, M., Turner, J., \& Balm, S. P. 1997, ApJ, 474, 741

Jura, M., \& Kahane, C. 1999, ApJ, 521, 302

Lopez, B., Mékarnia, D., \& Lefèvre, J. 1995, A\&A, 296, 752

Mannings, V., Boss, A. P., \& Russell, S. S. (ed.) 2000, Protostars and Planets IV, chapt. II. (Tucson, USA: The University of Arizona Press)

Men'shchikov, A. B., Schertl, D., Tuthill, P. G., Weigelt, G., \& Yungelson, L. R. 2002, A\&A, 393, 867

Roddier, F., Roddier, C., Graves, J. E., \& Northcott, M. J. 1995, ApJ, 443, 249

Sánchez Contreras, C., Desmurs, J. F., Bujarrabal, V., Alcolea, J., \& Colomer, F. 2002, A\&A, 385, L1

Schöier, F. L., \& Olofsson, H. 2001, A\&A, 368, 969

Soker, N. 2002, ApJ, 568, 726

Tuthill, P. G., Men'shchikov, A. B., Schertl, D., et al. 2002, A\&A, 389, 889

Waelkens, C., Van Winckel, H., Waters, L. B. F. M., \& Bakker, E. J. 1996, A\&A, 314, L17

Waters, L. B. F. M., Trams, N. R., \& Waelkens, C. 1992, A\&A, 262, L37

Zijlstra, A. A., Chapman, J. M., te Lintel Hekkert, P., et al. 2001, MNRAS, 322, 280

Zweigle, J., Neri, R., Bachiller, R., Bujarrabal, V., \& Grewing, M. 1997, A\&A, 324, 624 